\title{
The Corynebacterium pseudotuberculosis in silico predicted pan-exoproteome
}

\author{
Anderson R Santos ${ }^{1}$, Adriana Carneiro², Alfonso Gala-García', Anne Pinto', Debmalya Barh³, Eudes Barbosa', \\ Flávia Aburjaile', Fernanda Dorella', Flávia Rocha', Luis Guimarães ${ }^{1}$, Meritxell Zurita-Turk', Rommel Ramos², \\ Sintia Almeida', Siomar Soares', Ulisses Pereira', Vinícius C Abreu', Artur Silva², Anderson Miyoshi', \\ Vasco Azevedo ${ }^{1 *}$
}

From X-meeting 2011 - International Conference on the Brazilian Association for Bioinformatics and Computational Biology

Florianópolis, Brazil. 12-15 October 2011

\begin{abstract}
Background: Pan-genomic studies aim, for instance, at defining the core, dispensable and unique genes within a species. A pan-genomics study for vaccine design tries to assess the best candidates for a vaccine against a specific pathogen. In this context, rather than studying genes predicted to be exported in a single genome, with pangenomics it is possible to study genes present in different strains within the same species, such as virulence factors. The target organism of this pan-genomic work here presented is Corynebacterium pseudotuberculosis, the etiologic agent of caseous lymphadenitis (CLA) in goat and sheep, which causes significant economic losses in those herds around the world. Currently, only a few antigens against CLA are known as being the basis of commercial and still ineffective vaccines. In this regard, the here presented work analyses, in silico, five $C$. pseudotuberculosis genomes and gathers data to predict common exported proteins in all five genomes. These candidates were also compared to two recent C. pseudotuberculosis in vitro exoproteome results.

Results: The complete genome of five C. pseudotuberculosis strains (1002, C231, 119, FRC41 and PAT10) were submitted to pan-genomics analysis, yielding 306, 59 and 12 gene sets, respectively, representing the core, dispensable and unique in silico predicted exported pan-genomes. These sets bear 150 genes classified as secreted (SEC) and 227 as potentially surface exposed (PSE). Our findings suggest that the main C. pseudotuberculosis in vitro exoproteome could be greater, appended by a fraction of the 35 proteins formerly predicted as making part of the variant in vitro exoproteome. These genomes were manually curated for correct methionine initiation and redeposited with a total of 1885 homogenized genes.

Conclusions: The in silico prediction of exported proteins has allowed to define a list of putative vaccine candidate genes present in all five complete C. pseudotuberculosis genomes. Moreover, it has also been possible to define the in silico predicted dispensable and unique C. pseudotuberculosis exported proteins. These results provide in silico evidence to further guide experiments in the areas of vaccines, diagnosis and drugs. The work here presented is the first whole C. pseudotuberculosis in silico predicted pan-exoproteome completed till today.
\end{abstract}

\footnotetext{
* Correspondence: vasco@icb.ufmg.br

${ }^{1}$ Molecular and Celular Genetics Laboratory, Instituto de Ciências Biológicas,

Universidade Federal de Minas Gerais, Belo Horizonte, Minas Gerais, Brazil

Full list of author information is available at the end of the article
} 


\section{Background}

Reverse Vaccinology (RV) [1] analyses the genome sequence of a pathogen, which is an expected coded sequence for all the possible expressed genes in the pathogen's life cycle. All Open Reading Frames (ORF's) derived from the genome sequence can be evaluated using a computer program to determine their ability as vaccine candidates, giving special attention to exported proteins, as these are essential in host-pathogen interactions. Examples of such interactions include: (i) adherence to host cells, (ii) invasion of the cell to which there is compliance, (iii) damage to host tissues, (iv) environmental stresses resistance from the defense machinery of the cell being infected, and (v) mechanisms for subversion of host immune response [2-5].

Regarding exported proteins, these can distinguish between those that are exported to the cell wall, and after cleaved, release the mature portion into the extracellular milieu, which are referred to as secreted proteins (SEC), and those proteins exported to the cell wall which, even after cleaved, do not release the mature portion to the extracellular milieu, due to one or more hydrophobic motifs causing anchoring to the cell wall, and which are referred to as potentially surface exposed proteins (PSE). Different PSE subcategories exist according to the presence of a carboxy $(\mathrm{C})$ or amino $(\mathrm{N})$ terminal portion anchored to the cell wall, lipoproteins (E), end terminal loops (L), retention signals-like such as LGxTG, LysM, GW, Choline binding and PG binding (R), in combination or not with other PSE subcategories [6].

The term 'Reverse' from RV can be explained by the reverse genetics (RG) technique. Before the dawn of genomic, there were attempts to discover the responsible genes from a phenotype, reversing the research path of Crick's Central Dogma [7] (DNA $\rightarrow$ RNA $\rightarrow$ Protein) discovery. Holding the likely gene sequence, several techniques can be used to identify gene sequence modifications responsible for changes in the organism's phenotype. Crick's Central Dogma principle is also used for RV, as this technique searches within a gene sequence for possible proteins that could act as antigens capable of stimulating an immune response in a host organism [8].

The concept of RV was adapted to fit a new reality of widespread availability of genomic data [9]. With this technique, instead of searching for targets in a single strain or subspecies of an organism, it is now possible to simultaneously research in dozen of genomes, exploring potential joint antigens or exclusive ones to multiple genomes [10]. The availability of a large number of genomes to implement RV has lead to the emergence of the pangenomics reverse vaccinology concept [11], which can also apply to the concepts of core, extended (dispensable) and character (unique) genomes. While the core genome is composed of exported genes (genes that transcribe for exported proteins) that are common to these multiple strains and could represent candidates for a vaccine, the dispensable genome consists of genes that are absent in at least one of the strains of the studied species and the unique genome consists of genes that are specific to only a particular a strain [10]. From the standpoint of vaccines, the core genome represents to be a good candidate to compose a vaccine that is suitable for all studied strains. In this regard, the first step to enable any pangenomic reverse vaccinology study is to predict the core genome, along this work denominated in silico predicted pan-exoproteome (ISPPE). The model organism here analyzed (C. pseudotuberculosis) is a Gram-positive (GRAM+) bacterium, intracellular facultative parasite that affects small ruminants causing a chronic infectious pyogranulomatous disease characterized by the formation of abscesses in lymph nodes [12]. This pathogen infects mainly goats and sheep causing caseous lymphadenitis, but can also infect a huge variety of hosts throughout the world such as camels, horses, cattle, buffaloes, llamas, alpacas and, more rarely, humans [13-18], causing different diseases with different degrees of severity in each of them $[12,19]$.

\section{Results and discussion}

\section{In silico exoproteome prediction schema}

As shown in our proposed prediction schema (Figure 1), the software SurfG+ (Surface Gram positive), specially configured for GRAM+ bacteria, is responsible for most of the sub-cellular classifications, which vary between cytoplasmic (CYT), membrane (MEM), SEC and PSE (Figure 2). SurfG+ was configured for GRAM+ bacteria. Figure 1 represents the prediction schema using SurfG+ and three additional software, TatP 1.0 [20], SecretomeP 2.0 [21] and NclassG+ [22], which are specialized in nonclassical secretion prediction. SurfG + incorporates SignalP 3.0 predictor, responsible for identification of classical putative secreted proteins or exported proteins by the SEC pathway [23].

The results obtained after running SurfG+, TapP, SecretomeP and NClassG+ have gave rise to two gene data sets labeled as SEC and PSE, which correspond to the C. pseudotuberculosis ISPPE. These ISPPE data sets are composed of putative proteins present fivefold $(5 \mathrm{x})$, fourfold (4x), threefold (3x), twofold (2x) or onefold (1x), where fivefold means that a gene was predicted in all five strains, four fold meaning that a gene was predicted in four strains, and so on. A gene fold was obtained by reciprocal blast results, as described in the methods section. Since not all predicted genes are named, it was necessary to create a pan genome identifier, here denominated pan locus, to nominate each unique gene fold. The pan locus is unique within a pan genome and is shared by all homologous genes. For example, when a putative exported protein 


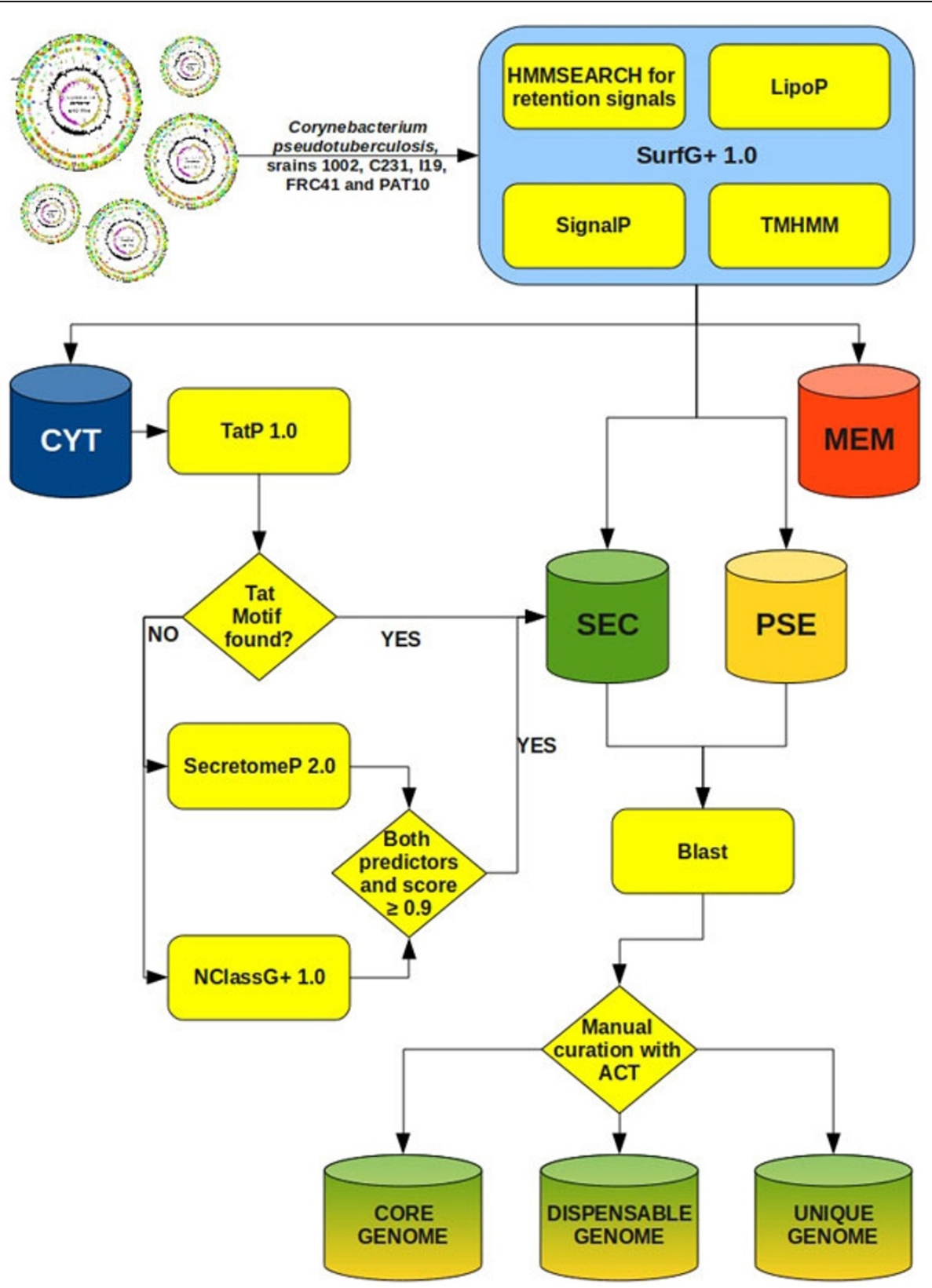

Figure 1 C. pseudotuberculosis pan genomic prediction schema. Software used, identified sub-cellular compartments and flow scheme to create the final pan genomic data sets.

was found within the five strains, each gene copy received the same pan locus to facilitate further data processing and identification. Following, it was necessary to confirm these results by systematical manual curation of each gene using the ACT tool from the Artemis software package [24]. Once completed this manual curation, it was possible to answer several questions regarding the correctness of each blast result and, as a consequence, it was possible to identify, for instance, that a gene formerly classified as $1 \mathrm{x}$ was indeed a $5 x$, as the other four gene copies were created starting beyond the signal peptide motif. After initial methionine correction, and also taking into account homologous genes, a new prediction step indicated all remaining putative proteins to be exported, composing the core ISPPE. However, gene's start positions incorporating a less probable signal peptide motif were also observed. In general, genes formerly predicted as $\mathrm{Nx}$ proved to be correct by manual curation as the remaining $(5-\mathrm{N}) \mathrm{x}$ genes were predicted as cytoplasmic, PSE or pseudogenes. These results are particularly interesting because they compose 


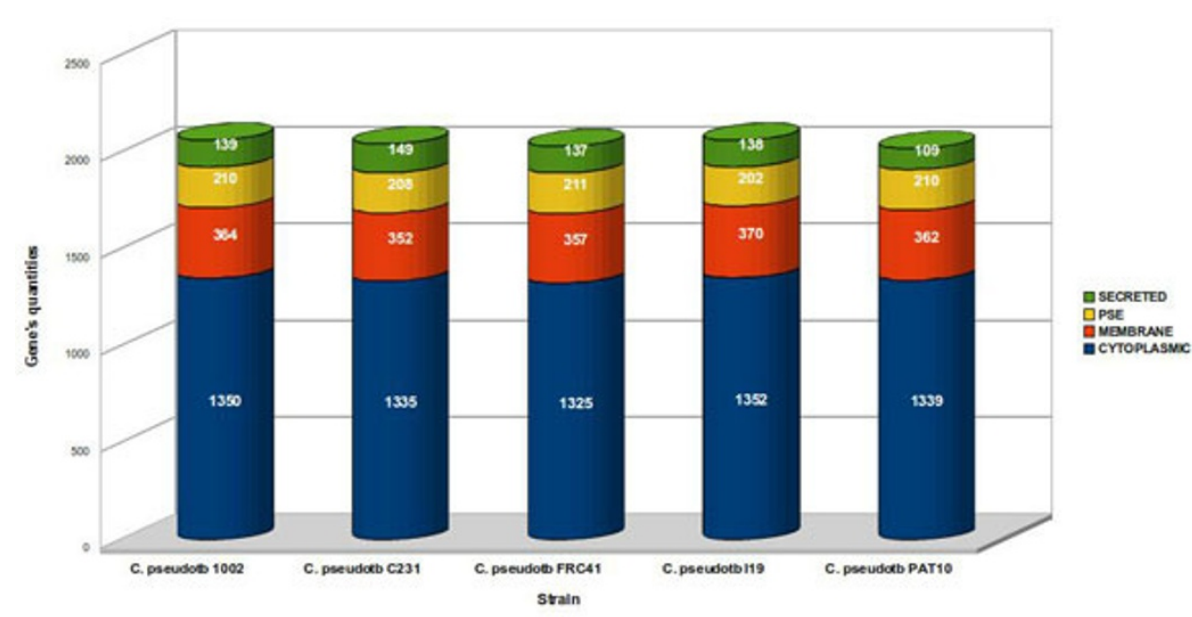

Figure 2 Predicted gene quantities by sub-cellular compartment from full C. pseudotuberculosis genomes. Classification of more than 10,000 distinct genes from the five different C. pseudotuberculosis strains in the four sub-cellular categories: cytoplasmic (CYT), membrane (MEM), potentially surface exposed (PSE) and secreted (SEC). Predictions were made using the schema presented in Figure 1.

the dispensable and unique ISPPE data sets. These genome annotation corrections, as a consequence of these analyses, were incorporated into the official annotation of the five C. pseudotuberculosis strains deposited at GenBank in August, 2011. This genomes are also available in the additional file 1, as EMBL files.

\section{Classical and non-classical secreted putative proteins}

Figure 3 exhibits the in silico predicted pan secretome results for C. pseudotuberculosis, which comprise 150 genes, out of 377 from the whole ISPPE, representing 750 locus_tags in the five studied C. pseudotuberculosis strains.
However, despite representing 750 locus_tags, not all were predicted as secreted. If at least one gene copy, within a specific pan locus, was not predicted as secreted, it still received the same pan locus but was not classified as part of the predicted core secretome. There are 122 genes composing the predicted core secretome ( $5 \mathrm{x})$, followed by 25 genes constituting the predicted dispensable secretome $(4 \mathrm{x}, 3 \mathrm{x}$ and $2 \mathrm{x})$ and just 3 genes as the predicted unique secretome (1x). These results were obtained applying the prediction schema from Figure 1; however, different contributions were obtained from different predictors, as shown in Figure 4.

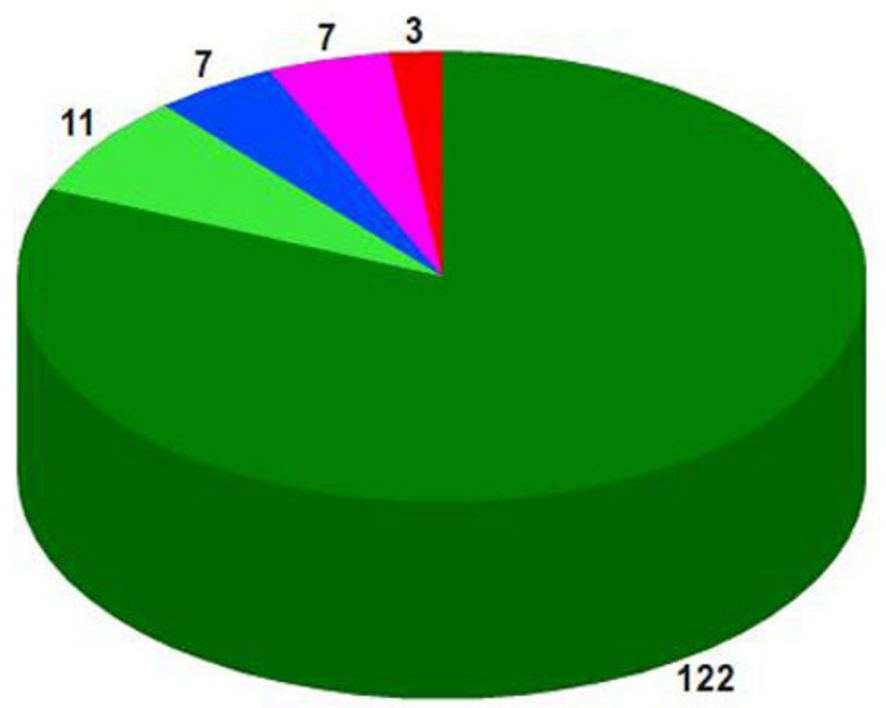

Figure 3 Predicted C. pseudotuberculosis pan secretome. Predictions for 150 genes from strains 1002, C231, I19, FRC41 and PAT10 made by SurfG+ 1.0, TatP 1.0 Server and SecretomeP 2.0 Server. 

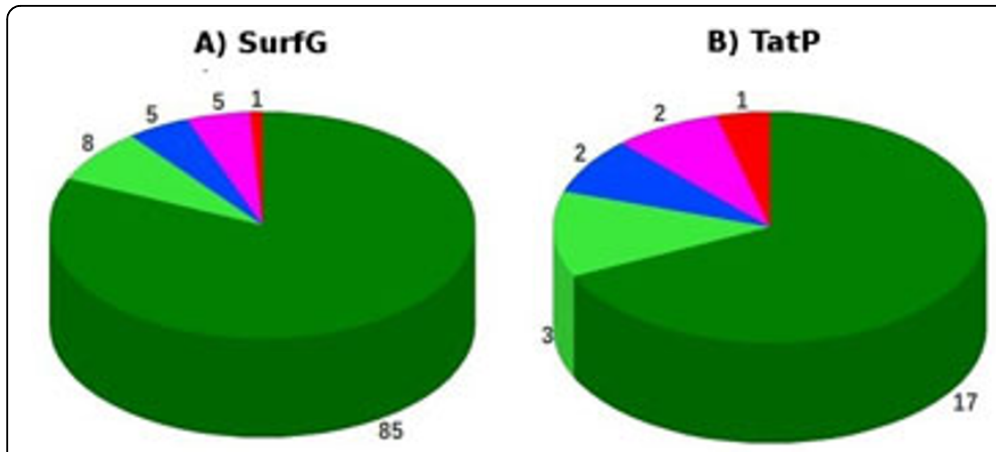

C) SecretomeP and NClassG+

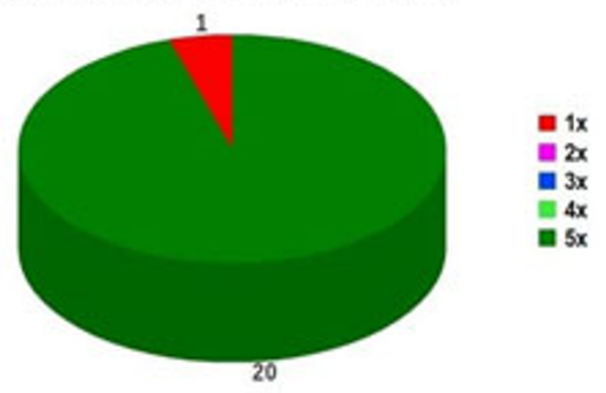

Figure 4 Predicted C. pseudotuberculosis pan secretome by predictor software. Predicted secreted genes coverage in the predicted pan secretome of the five bacterial strains separated by predictor software SurfG+, TatP and SecretomeP.

SurfG+ predicted 104 genes, corresponding 85,18 and 1 to the predicted core, dispensable and unique secretome respectively. On the other hand, TatP predicted 25 genes, of which 17, 7 and 1 corresponded to the predicted core, dispensable and unique secretome respectively. Finally, SecretomeP and NClassG+ predicted 21 genes, corresponding 20 and 1 to the predicted core and unique secretome respectively. It can be easily observed that the main predicted portion is originated by SurfG+, as it predicts putative proteins possibly secreted by the SEC pathway. A considerable portion of genes $(\sim 31 \%)$, only within the predicted core secretome, comes from non-classical secretion predictors that cannot be ignored when the subject is about vaccine candidates.

The dispensable and unique C. pseudotuberculosis predicted secretomes contain $\sim 8 \%$, or 58 locus_tags, not predicted as secreted. Putative proteins predicted as CYT, PSE and putative frame shifts (pseudogenes) account for 22, 24 and 10 locus_tags respectively. In the dispensable and unique C. pseudotuberculosis in silico predicted secretomes, the numbers of genes identified as membrane integral or absent in a genome are insignificant. Nevertheless, the manual curation step ensured no annotation errors in these predictions, making it possible to claim the hypothesis that these differences could be due to environment adaptations. A table containing the complete list of C. pseudotuberculosis secreted proteins is available in the additional file 2.

\section{Potentially surface exposed (PSE) putative proteins}

The SurfG + software was calibrated by the cell wall thickness for each C. pseudotuberculosis strain. Figure 5 shows 184 genes, out of 377 from the whole ISPPE, comprising the predicted core surfaceome (5x), 34 genes composing the predicted dispensable surfaceome $(4 x, 3 x$ and $2 x)$ and just 9 genes as predicted unique surfaceome (1x). These 227 genes account for 1135 locus_tags in all five strains. In this set, homologous genes within a pan locus do not ever share the same sub-cellular prediction. Genes predicted as
MEM, CYT, SEC and putative pseudogenes account for 29, 23, 20 and 17 distinct locus_tags, respectively. Genes predicted as MEM ( 3\%) compose the second major group. This could be explained by the fact that membrane proteins already contain hydrophobic extension and could be more susceptible to expose or occult parts of a protein to the extracellular milieu. However, the same reasoning does not suit to explain the third major group of locus_tags with surfaceome pan locus that correspond to proteins predicted as secreted ones. These 20 locus_tags that were predicted as secreted, but also received surfaceome pan locus, raise a question; do these fit SEC or PSE labels? There exist no simple paths to estimate their sub-cellular compartment by software, since some locus_tags were predicted as PSE receiving surfaceome pan locus and other were predicted as SEC and also received secretome pan locus. Ten pan locus (plcppse193, plcppse194, plcppse205, plcppse218, plcppse226, plcpsec096, plcpsec097, plcpsec098, plcpsec100, plcpsec101) faces this question, as some genes appear in both the predicted secretome and surfaceome.

The PSE subcategories show predominance of genes, as presented in Figure 6. Most of the 1045 genes predicted as PSE are cell wall anchored outward C-terminal $(\sim 40 \%)$ ( $\geq 50$ AA long), followed by lipoproteins ( $24 \%)$, outward loops $(\sim 11 \%)$ ( $\geq 100$ AA long) and outward N-terminal ( $17 \%)$ ( $\geq 50$ AA long), whereas genes containing retention signals (PSE R) account only for $\sim 8 \%$.

The PSE results of all strains were analyzed considering that a significant cell wall thickness difference between strain I19 and the other ones was observed ( 34 nm versus $\sim 24 \mathrm{~nm}$ ). Despite the significant cell wall thickness difference, a small difference was predicted in the genome, which accounts for a decrease in the number of PSE and an increase in the number of MEM genes in C. pseudotuberculosis strain I19. A table containing the complete list of C. pseudotuberculosis PSE proteins is available in the additional file 3. 


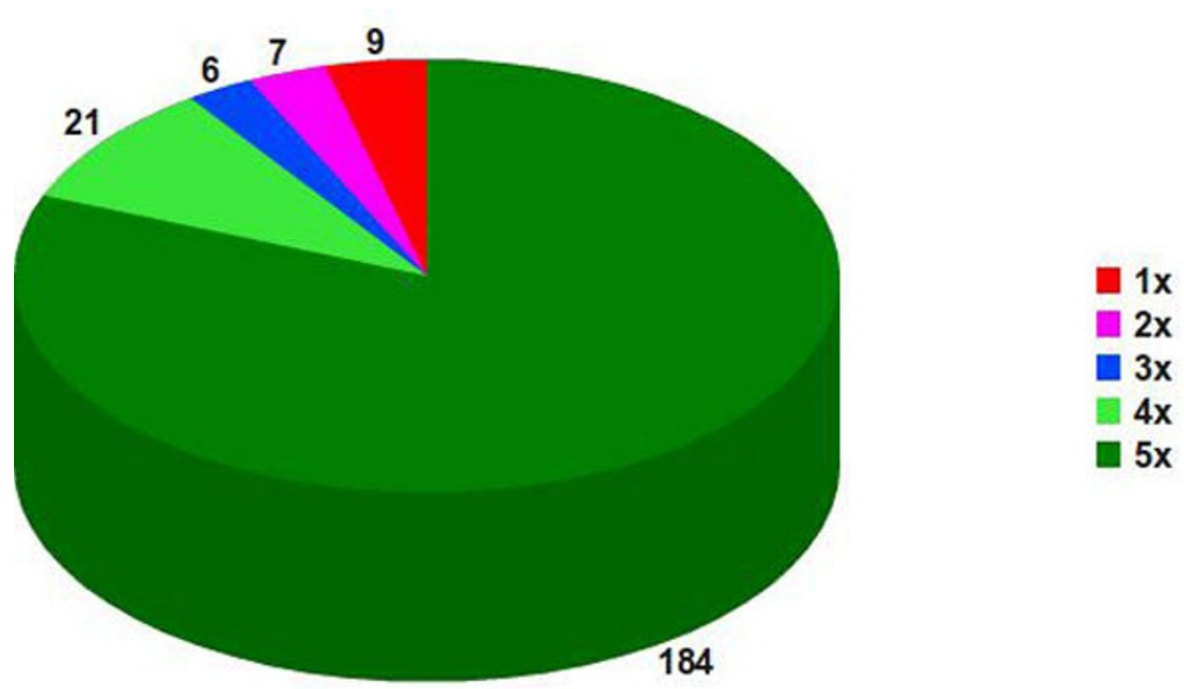

Figure 5 Predicted C. pseudotuberculosis pan surfaceome. Pan surfaceome predictions for 227 genes from strains 1002, C231, 119, FRC41 and PAT10, performed by SurfG + 1.0.

\section{Revised in vitro exoproteome results}

The 104 observed genes in both TPP/LC-MS ${ }^{\mathrm{E}}$ [25] and 2DE-MALDI-TOF/TOF, (Silva WM, Seyffert N, Castro TLP, Santos AV, Pacheco LGC, Santos AR, Ciprandi A, Zurita-Turk M, Dorella FA, Andrade HM, Pimenta AMC, Silva A, Miyoshi A, Azevedo V, unpublished observations) experiments were compared with the ISPPE results here presented. This comparison, explained in the methods section, brought novel insights into the in vitro exoproteome and showed the possibility of having additional genes in the main C. pseudotuberculosis in vitro exoproteome. In Table 1 are listed all 35 proteins of the variant in vitro exoproteome (strains 1002 and C231), that correspond to $\sim 23 \%$ of the total amount. These proteins were found to be highly conserved in the five compared C. pseudotuberculosis strains and comprise the core ISPPE. Moreover, it was verified that three proteins (ADL20466, ADL20097 e ADL19973), previously classified as belonging to the variant in vitro exoproteome of strains 1002 [25], did actually belong to the main in vitro exoproteome. These findings

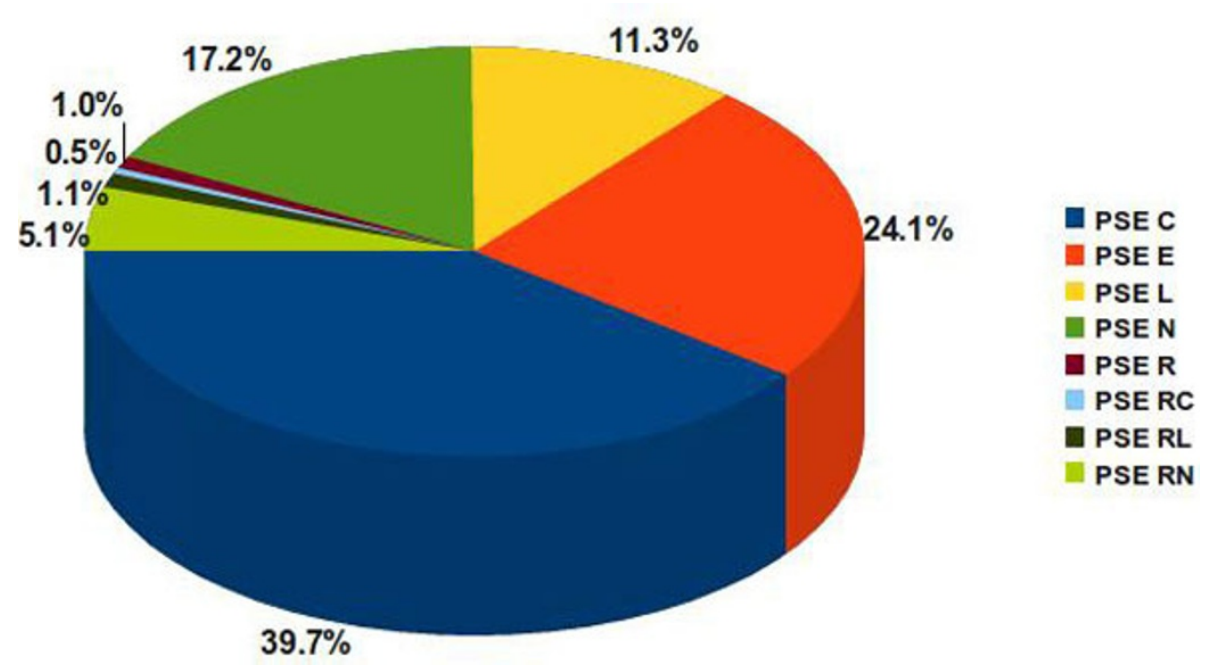

Figure 6 Predicted C. pseudotuberculosis pan surfaceome by PSE subcategories. PSE categories are distributed in outward C-terminal or Nterminal portion greater than or equal 50 AA. Outward N or C terminal greater than 100 AA are classified as L. Lipogenes identified by LipoP are classified as $\mathrm{E}$ and retention signals identified by HMMSEARCH profiles are classified as R. These labels can also be conjugated to create other PSE subcategories. 
Table 1 Core C. pseudotuberculosis in silico predicted pan-exoproteome found in the variant in vitro exoproteome

\begin{tabular}{|c|c|c|c|c|c|}
\hline $\begin{array}{l}\text { Protein } \\
\text { identifier }\end{array}$ & locus_tag & $\begin{array}{l}\text { Gene } \\
\text { name }\end{array}$ & Product & $\begin{array}{l}\text { Predicted local sub- } \\
\text { cellular }\end{array}$ & $\begin{array}{c}\text { GenBank organism } \\
\text { identifier }\end{array}$ \\
\hline ADL19972 & Cp1002_0064 & & Hypothetical protein & PSE E & CP001809 \\
\hline ADL20140 & Cp1002_0237 & $\operatorname{sipA}$ & Surface layer protein $\mathrm{A}$ & SEC & CP001809 \\
\hline ADL20222 & Cp1002_0320 & & Hypothetical protein & PSE N & CP001809 \\
\hline ADL20288 & Cp1002_0388 & & L,D-transpeptidase catalytic domain, region YkuD & SEC & CP001809 \\
\hline ADL20391 & Cp1002_0497 & male & $\begin{array}{l}\text { Maltose/maltodextrin transport system substrate- } \\
\text { binding protein }\end{array}$ & PSE E & CP001809 \\
\hline ADL20455 & Cp1002_0562 & sprT & Trypsin & PSE C & CP001809 \\
\hline ADL20477 & Cp1002_0584 & cynt & Carbonic anhydrase & PSE E & CP001809 \\
\hline ADL20508 & Cp1002_0615 & & Hypothetical protein & SEC & CP001809 \\
\hline ADL20574 & Cp1002_0681 & $r p f B$ & Resuscitation-promoting factor RpfB & SEC & CP001809 \\
\hline ADL20656 & Cp1002_0766 & & Hypothetical protein & SEC & CP001809 \\
\hline ADL21028 & Cp1002_1144 & yceG & Amino deoxychorismate lyase & SEC & CP001809 \\
\hline ADL21239 & Cp1002_1362 & & Hypothetical protein & PSE E & CP001809 \\
\hline ADL21302 & Cp1002_1425 & $\mathrm{ctaC}$ & Cytochrome c oxidase subunit II & PSE C & CP001809 \\
\hline ADL21537 & Cp1002_1669 & & Hypothetical protein & SEC & CP001809 \\
\hline ADL21667 & Cp1002_1802 & lipY & Secretory lipase & SEC & CP001809 \\
\hline ADL09524 & CpC231_0025 & pld & Phospholipase D & SEC & CP001829 \\
\hline ADL09532 & CpC231_0033 & $p b p A$ & Penicillin-binding protein A & SEC & СР001829 \\
\hline ADL09691 & CpC231_0196 & & Hypothetical protein & SEC & CP001829 \\
\hline ADL09697 & CpC231_0203 & $p b p B$ & Penicillin binding protein transpeptidase & SEC & СР001829 \\
\hline ADL09852 & CpC231_0360 & oppA1 & Oligopeptide-binding protein oppA & PSE E & CP001829 \\
\hline ADL09871 & CpC231_0379 & & Hypothetical protein & SEC & CP001829 \\
\hline ADL09872 & CpC231_0380 & male & Maltotriose-binding protein & PSE E & CP001829 \\
\hline ADL09990 & CpC231_0503 & lytR & Transcriptional regulator lytR & PSE C & CP001829 \\
\hline ADL10248 & CpC231_0766 & & Hypothetical protein & SEC & CP001829 \\
\hline ADL10460 & CpC231_0982 & $\operatorname{cin} A$ & Iron $\mathrm{ABC}$ transporter substrate-binding & PSE E & CP001829 \\
\hline ADL10489 & CpC231_1012 & ycel & Protein ycel & SEC & СР001829 \\
\hline ADL10626 & CpC231_1150 & & Zinc metallopeptidase & PSE C & CP001829 \\
\hline ADL10663 & CpC231_1187 & & Lipoprotein & PSE E & CP001829 \\
\hline ADL10880 & CpC231_1409 & $p k n L$ & Serine/threonine protein kinase & PSE N & CP001829 \\
\hline ADL11196 & CpC231_1737 & & Corynomycolyl transferase & SEC & СР001829 \\
\hline ADL11213 & CpC231_1756 & & Hypothetical protein & SEC & CP001829 \\
\hline ADL11326 & CpC231_1871 & & Hypothetical protein & PSE N & CP001829 \\
\hline ADL11338 & CpC231_1885 & & Membrane protein & SEC & CP001829 \\
\hline ADL11339 & CpC231_1886 & & Hypothetical protein & SEC & CP001829 \\
\hline ADL11410 & CpC231_1959 & $g / p Q$ & Glycerophosphoryl diester phosphodiesterase & PSE E & CP001829 \\
\hline
\end{tabular}

The 35 proteins listed in this table were not found in the experimental main in vitro exoproteome $[47 ; 48]$ but were found in the in silico predicted panexoproteome of all five C. pseudotuberculosis strains.

give raise to the possibility that more proteins of the variant in vitro exoproteome indeed make part of the main in vitro exoproteome.

This comparison also served as a rebuttal argument against some specific genes. The Cp1002_0369 gene, classified under the plcpsec100 pan locus as a pseudogene, was identified by the in vitro exoproteome experiment. Interestingly, this gene copy also suits the plcppse226 pan locus. Both pan locus make part of previous related genes that already showed difficulties to be classified, by software, into any potential sub-cellular compartment, as some genes within the pan locus fit both SEC and PSE labels. The in silico predictions enforces that there are at least three secreted proteins, inspite of the other two gene copies being predicted as having PSE and CYT labels. 
Furthermore, the genes plcppse180, plcppse192, plcpsec077, plcpsec095 and plcpsec099 also had both genes found in the main in vitro exoproteome of strains 1002 and C231, but were not classified in the ISPPE. The plcppse180 pan locus holds a putative pseudogene (CpPAT10_0459), and is therefore not present in the in silico predicted core surfaceome. Other genes were predicted as cytoplasmic. It is possible that these genes were wrongly assembled since there is evidence that at least two homologous genes, from strains 1002 and C231, are exported to the extracellular milieu.

\section{Core C. pseudotuberculosis ISPPE candidates homologous to $M t b$}

Within the core C. pseudotuberculosis ISPPE, homologous genes to those of the previously studied Mycobacterium tuberculosis H37Rv (Mtb) were observed. In this work we present some of these homologous genes featuring at least $90 \%$ protein alignment and $50 \%$ identity within this alignment. These cut-offs were obtained during the search for C. pseudotuberculosis homologous genes in the $M t b$ genome.

The core $C$. pseudotuberculosis ISPPE, that accounts for $\sim 81 \%$ of the total, is composed of 306 genes or 1,530 distinct locus_tags, being 40\% predicted as SEC and $~ 60 \%$ predicted as PSE proteins, of which 20 genes present high similarity to $M t b$ 's genes (Table 2); however, not all of these $M t b$ genes have known functions.

In this regard, here we only discuss some of these $M t b$ 's genes with experimental evidence. The plcppse174 pan locus shows $51 \%$ protein identity with Rv3915 (YP_178027.1), a gene named $c w l M$ that was the first autolysin gene identified and cloned from $M t b$. This finding offers a new drug target class that could alter the permeability of the mycobacterium cell wall and enhance the effectiveness of treatments for tuberculosis [26]. Applying principles of in vivo expression technology (IVET), it was possible to identify upregulated genes from $M t b$ in an in vitro simulation of anaerobic persistence condition. The upregulated genes under hypoxic condition (dissolved oxygen $<1 \%$ ) include Rv0050 (ponA1), a penicillin binding protein that has $52 \%$ protein identity to the plcppse165 pan locus and 90\% alignment extension [27]. The plcpsec 122 pan locus shows $\sim 58 \%$ protein identity with Rv2752c (NP_217268.1), a unique bi-functional $M t b$ gene that owns both $\beta$-lactamase and RNase activities. Both activities are lost upon deletion of the 100 AA long C-terminal 100 tail, which contains an additional loop when compared to the RNase J of Bacillus subtilis [28]. As it can be observed, the plcppse080 pan locus appears twice in Table 2, as it is homologous to both NADH dehydrogenase gene copies of $M t b, n d h$ (NP_216370.1) and $n d h A$ (NP_214906.1), with $\sim 57 \%$ protein identity. In $M t b$, energy generation is mainly performed by type II dehydrogenases $n d h$ and $n d h A$, being both, as such, essential genes [29].

The plcpsec113 pan locus is homologous to the $g \operatorname{lm} U$ gene (NP_215534.1), holding 59\% protein identity and more than $90 \%$ alignment extension. This gene is essential in $M t b$, being required for optimal bacterial growth, and has been selected as a possible drug target for structural and functional investigation [30]. GlmU is a bifunctional acetyltransferase/uridyltransferase that catalyses the formation of UDP-GlcNAc from GlcN-1-P. UDP-GlcNAc is the substrate for two important biosynthetic pathways: lipopolysaccharide and peptidoglycan synthesis. Due to its important roles, $g \operatorname{lm} U$ had its conformational structure solved [30]. The plcpsec113 pan locus for C. pseudotuberculosis is an interesting putative drug candidate since it is predicted to be secreted, part of the core ISPPE and is able to infer its conformational structure by homology modeling using $M t b \operatorname{glm} U$.

Several genes involved in mannoglycoconjugate biosynthesis have shown to be involved in virulence, due to their central role in biosynthesis of major surface-associated glycoconjugates. Within these genes, the $M t b$ gene $\operatorname{manB}$ (Rv3264c) is defined as a GDP-mannose pyrophosphorylase (GDPMP) and disruption of its activity leads to decrease of surface-associated mannosylated lipoglycans. For GDPMP, this decrease correspond directly to reduced virulence in both BALB/c mice and cultured human macrophages [31]. The $M t b \operatorname{manB}$ gene holds $69 \%$ protein identity to the plcpsec 110 pan locus and more than $90 \%$ alignment extension, making plcpsec 110 a considerable putative drug target.

Mycolic acids and multimethyl-branched fatty acids are found uniquely in the cell envelope and are essential for survival, virulence and antibiotic resistance of $M t b$. AcylCoA carboxylases (ACCases) commit acyl-CoAs to the biosynthesis of these unique fatty acids. Previous studies indicate that AccD5 is important for cell envelope lipid biosynthesis and its disruption leads to pathogen death [32]. The $M t b$ gene $a c c D 5$ (NP_217797.1) had its structure determined and also shows $\sim 74 \%$ protein identity to the plcppse 045 pan locus in more than $90 \%$ alignment extension, making it also a promising candidate for further vaccine candidate evaluations.

Moreover, it was demonstrated that $M t b$ can use heme as an iron source, suggesting that $M t b$ contains a yetunknown heme acquisition system [33]. We found that the C. pseudotuberculosis plcpsec076 pan locus holds $\sim 52 \%$ protein identity to the $M t b$ gene hemE (NP_217194.1) and more than $90 \%$ alignment size, therefore also representing an interesting drug target for C. pseudotuberculosis.

\section{Candidates filtering}

The here presented results provide a plethora of putative vaccine candidates never seen before for 
Table 2 Core C. pseudotuberculosi s in silic o predicted pan-exoproteome homologous to Mtb's proteins

\begin{tabular}{|c|c|c|c|c|c|c|c|c|}
\hline \multicolumn{3}{|c|}{$\begin{array}{l}\text { Corynebacterium } \\
\text { pseudotberculosis }\end{array}$} & \multirow[b]{2}{*}{$\begin{array}{l}\% \text { of amino acid } \\
\text { alignment's } \\
\text { identity }\end{array}$} & \multicolumn{5}{|c|}{$\begin{array}{l}\text { Mycobacterium } \\
\text { tuberculosis }\end{array}$} \\
\hline pan locus & $\begin{array}{l}\text { Reference } \\
\text { genome } \\
\text { locus_tag }\end{array}$ & $\begin{array}{l}\text { ORF } \\
\text { size }\end{array}$ & & $\begin{array}{l}\text { ORF } \\
\text { size }\end{array}$ & locus_tag & $\begin{array}{l}\text { Gene } \\
\text { name }\end{array}$ & protein ID & Annotated product \\
\hline plcpsec106 & cpfrc_00104 & 488 & 69.10 & 461 & Rv3790 & & NP_218307.1 & oxidoreductase \\
\hline plcpsec076 & cpfrc_00276 & 371 & 51.56 & 357 & Rv2678c & hemE & NP_217194.1 & uroporphyrinogen decarboxylase \\
\hline plcppse023 & cpfrc_00283 & 535 & 52.51 & 529 & Rv0528 & & NP_215042.1 & transmembrane protein \\
\hline plcppse045 & cpfrc_00491 & 543 & 73.72 & 548 & Rv3280 & accD5 & NP_217797.1 & propionyl-CoA carboxylase beta chain \\
\hline plcpsec110 & cpfrc_00506 & 362 & 69.03 & 359 & Rv3264c & $\operatorname{man} B$ & YP_177951.1 & $\begin{array}{l}\text { D-alpha-D-mannose-1-phosphate } \\
\text { guanylyltransferase MANB }\end{array}$ \\
\hline plcpsec111 & cpfrc_00508 & 151 & 51.45 & 139 & Rv3259 & & NP_217776.1 & hypothetical protein \\
\hline plcpsec113 & cpfrc_00705 & 487 & 58.67 & 495 & Rv1018c & $\mathrm{g} / \mathrm{mU}$ & NP_215534.1 & $\begin{array}{c}\text { bifunctional N-acetylglucosamine-1-phosphate } \\
\text { uridyltransferase/glucosamine-1-phosphate } \\
\text { acetyltransferase }\end{array}$ \\
\hline plcpsec115 & cpfrc_00945 & 64 & 63.33 & 64 & Rv1642 & rpml & NP_216158.1 & $50 S$ ribosomal protein $\mathrm{L} 35$ \\
\hline plcppse080 & cpfrc_01015 & 452 & 57.08 & 470 & Rv0392c & $n d h A$ & NP_214906.1 & membrane NADH dehydrogenase \\
\hline plcppse080 & cpfrc_01015 & 452 & 58.10 & 463 & Rv1854c & ndh & NP_216370.1 & NADH dehydrogenase \\
\hline plcpsec041 & cpfrc_01074 & 403 & 62.96 & 381 & Rv1488 & & NP_216004.1 & hypothetical protein \\
\hline plcpsec119 & cpfrc_01121 & 504 & 53.71 & 457 & Rv1407 & $f m u$ & NP_215923.1 & Fmu protein (SUN protein) \\
\hline plcppse085 & cpfrc_01126 & 417 & 55.58 & 418 & Rv1391 & $d f p$ & NP_215907.1 & $\begin{array}{l}\text { bifunctional phosphopantothenoylcysteine } \\
\text { decarboxylase/phosphopantothenate synthase }\end{array}$ \\
\hline plcpsec138 & cpfrc_01214 & 79 & 68.42 & 82 & Rv2708c & & NP_217224.1 & hypothetical protein \\
\hline plcpsec122 & cpfrc_01267 & 683 & 57.76 & 558 & Rv2752c & & NP_217268.1 & hypothetical protein \\
\hline plcpsec124 & cpfrc_01393 & 239 & 57.83 & 250 & Rv2149c & yfiH & NP_216665.1 & hypothetical protein \\
\hline plcppse104 & cpfrc_01424 & 412 & 50.38 & 429 & Rv2195 & qcrA & NP_216711.1 & Rieske iron-sulfur protein QcrA \\
\hline plcpsec128 & cpfrc_01757 & 313 & 59.42 & 322 & Rv3579c & & NP_218096.1 & tRNA/rRNA methyltransferase \\
\hline plcppse131 & cpfrc_01798 & 480 & 62.21 & 491 & Rv2443 & $d c t A$ & NP_216959.1 & $\begin{array}{c}\text { C4-dicarboxylate-transport transmembrane protein } \\
\text { DctA }\end{array}$ \\
\hline plcppse165 & cpfrc_02038 & 721 & 52.00 & 678 & Rv0050 & ponA1 & YP_177687.1 & bifunctional penicillin-binding protein $1 \mathrm{~A} / 1 \mathrm{~B}$ \\
\hline plcppse174 & cpfrc_02102 & 393 & 51.41 & 406 & Rv3915 & CWIM & YP_178027.1 & hydrolase \\
\hline
\end{tabular}

Related C. pseudotuberculosis's proteins containing at least 50\% amino acid identity and 90\% alignment size to the Mtb H37Rv's proteins.

C. pseudotuberculosis. However, genes predicted as MEM and CYT account respectively for $18 \%$ and $65 \%$ of the in silico predicted pan genome. Despite the 227 surfaceome and 150 secretome genes here presented, these only represents $\sim 16 \%$ of the C. pseudotuberculosis in silico predicted pan genome. Most of the genes remain inaccessible for the current in silico prediction techniques and it is possible that these neglected genes could also be good candidates against $C$. pseudotuberculosis. These findings raise the need for more elaborated and driven software or prediction schemas capable of uncovering these major genome neglected portions. Using the prediction schema here presented, it was possible to include more than $\sim 2 \%$ of non-classic secreted putative proteins that compose putative vaccine candidates. However, this low income amount of vaccine candidates is due to the optional parameter selected in our prediction schema, the non-classic secreted score greater than or equal 0.90. If using the default parameter from the software secretomeP and
NClassG+, this income would be increased up to $\sim 6 \%$ and the final income of putative vaccine candidates would be $\sim 20 \%$, using a couple of motifs predictors as depicted in Figure 1. The current reverse vaccinology software allows obtaining a number of candidates closer to $20 \%$ of the C. pseudotuberculosis genome. These considerations raise a question: supposing that novel software for unexplored secretion pathways come into scenario, what is the genome's percentage that could be selected as putative vaccine candidates? Supposing that this percentage reaches $40 \%$, how could the problem of choosing between almost one thousand putative vaccine candidates to be used for the next vaccine production stage for C. pseudotuberculosis be solved? This dilemma could be solved by using further software prediction just like those addressing epitopes MHC class I and II allele affinity [34]; however, this could be just a part of the solution. There are chances of solving this dilemma by means of broader vaccine projects, which would take into account particular variables for 
each target organism in order to minimise research efforts and the number of possible vaccine candidates [35].

\section{In silico versus non-in silico}

It is broadly known that in silico genome investigations could give evidence about the genome's function and structure. It is also known that such in silico investigations could only be proved or denied by non-in silico experiments. Therefore, such reasonable thinking is not a singlehand avenue. Non-in silico experiments could be improved by means of more comprehensive or specific approaches with the objective of getting a closer answer to the reality for biological questions. The fact is that in silico analyses cannot vary when executed over and over again and no matter how many folds are run. We know that exactly 122 genes will be always predicted as having classical exportation motifs; on the other hand, we cannot expect the same behavior from non-in silico analysis. Some real proteins could be or not be found in an in vitro or in vivo exoproteome result, due to an uncountable number of factors [21]. Therefore, we suggest that the core C. pseudotuberculosis ISPPE could be composed of a larger number of predicted genes, but such confirmation could only be affirmed with additional non-in silico exoproteome experiments.

\section{Conclusions}

The in silico pan-exoproteome prediction methodology applied to the pathogen C. pseudotuberculosis helps to raise new insights into putative vaccine candidates against CLA. Additional investigations of the in vitro exoproteome of two strains of C. pseudotuberculosis, 1002 and C231, showed evidence that the major part of the variant in vitro exoproteome is contained in the core ISPPE. A simultaneous curation of the in silico predicted core secretome and surfaceome within the five C. pseudotuberculosis strains also contributed to homogenize the genome annotations and it was possible to fix the most probable putative methionine proteins. Moreover, putative miss assembled genes, formerly classified as pseudogenes by in silico analyses, were also revised. The efforts to create a C. pseudotuberculosis ISSPE catalogue proved to be necessary and computationally viable to ensure a uniform set of putative vaccine candidates free of annotation errors.

\section{Methods}

\section{Genomes}

The analyzed C. pseudotuberculosis genomes were obtained from the GenBank according to the following accession numbers: EMBL: CP001809 (strain 1002), EMBL: CP001809 (strain C231), EMBL: CP002251 (strain I19), EMBL: CP002924 (strain PAT10) and EMBL: NC_014329 (strain FRC41).

\section{Prediction schema}

Predicted genes from all five C. pseudotuberculosis strain genomes were exported as amino acid fasta files using the Artemis software. These fasta files were passed as parameters to SurfG+ 1.0 (Figure 1), and lists of genes predicted as CYT, SEC, PSE and MEM were created by this software. Genes formerly predicted as CYT by SurfG+ were then submitted to the TapP 1.0 predictor; when a Tat motif was found, the putative protein was automatically classified as SEC, otherwise, another prediction round would took place using two other non-classic secretion predictors, SecretomeP 2.0 and NclassG+ 1.0. With a positive prediction from both software and a prediction score greater than or equal to 0.90 , the genes were automatically classified as SEC. The SEC and PSE data sets were finally submitted to a reciprocal blastp processing and posterior filtering, giving rise to the fivefold categories according to folds occurring in each strain: $5 x, 4 x, 3 x, 2 x$ and $1 x$. The results were then manually curated using the ACT software and strain 1002, the first to be sequenced and annotated. The strain 1002 was disposed, in ACT software, in the middle of two pairs of the other two genome strains, facilitating to exhibit differences among all of them.

\section{SurfG +1.0}

Sub-cellular localization prediction of C. pseudotuberculosis putative proteins was made by in silico analysis using the SurfG+ 1.0 software [6]. SurfG+ is a pipeline for protein sub-cellular prediction that incorporates common software, such as SignalP, LipoP and TMHMM to search for motifs. It also creates novel HMMSEARCH profiles to predict cell wall retention signals. SurfG+ starts searching, in the following order for: retention signals, lipoproteins, SEC pathway export motifs and transmembrane motifs. If none of these motifs are found in a protein sequence, it is then characterized as CYT. A novel possible characterization introduced by SurfG+ is its ability to better distinguish between MEM and PSE, by informing an expected cell wall thickness in amino acids. Using the literature or an electronic microscopy it is possible to estimate a reasonable cell wall thickness value for prokaryotic organisms. By means of this last option, C. pseudotuberculosis genes were classified into four different sub-cellular locations: CYT, MEM, PSE, or SEC.

\section{TatP 1.0 Server}

Twin-arginine signal peptide motifs were predicted using the on line server hosted by http://www.cbs.dtu. $\mathrm{dk} /$ services/TatP/[20]. Only putative proteins formerly classified as CYT by SurfG + were submitted to the TatP analyses. There were no intersections between SignalP and TatP predictions. 


\section{SecretomeP 2.0 and NClassG +1.0}

Non-classical secreted putative proteins were predicted using the online server hosted by http://www.cbs.dtu.dk/ services/SecretomeP/[21]. NClassG+ [22], a second nonclassical secreted protein predictor, was also used; however, the predictions were directly performed contacting the software authors. This double check prediction ensured greater accuracy. Only those genes formerly classified as CYT by SurfG+ and without the twin-arginine signal peptide motifs were submitted to a non-classical secreted analysis. Despite the significant scores of SecretomeP and Nclass+, ranging between 0.5 and 1.0, only those genes with a score greater than or equal to 0.9 were selected, in order to ensure a minimal false positive in future wet lab experiments, the focus of our research group.

\section{Pan genome}

To predict the C. pseudotuberculosis pan genome, reciprocal blastp results were used. All the putative proteins predicted as SEC were put apart in a single amino acid fasta file to make a reciprocal blast. A similar file was also created for the proteins predicted as PSE. To avoid homologous mismatches, the blastp results obtained using the PAM70 substitution matrix and the $10^{-6} \mathrm{e}$-value were manually filtered. In this regard, the first step was to establish the alignment size and identity percentages of cut-offs, being 89.58 and $50.00 \%$, respectively, for SEC putative proteins, whereas for PSE putative proteins, these cut-offs were 88.16 and $48.80 \%$, respectively. Identity percentages closer to $50 \%$ are explained by frame shifts not annotated until this work. All the putative proteins from the five strains (query) with alignment size and identity percentages higher than these cut-offs had no more than one group of blast hits (subject) against the others strains. Moreover, within each of these blast hits groups, there was a blast hit from the query protein against it self as subject. The results were manually curated using the ACT software, from the Artemis package [24], using the strain 1002 as reference strain for the other two strains. This ACT view was composed by strains C231-1002-I19 and FRC41-1002-I19. Each putative protein predicted as SEC and PSE was compared against their other four homologues for correct initial methionine, frame shifts and finally annotating the correct sub-cellular location.

\section{Revised in vitro exoproteome results}

In lists 1 and 2 of the annex are both gene locus present in the C. pseudotuberculosis ISPPE, together with the quantity of homologous genes present in the all five genomes. These results were inserted in a relational database, denominated C. pseudotuberculosis Data Base (CpDB) [36], in a specific table called 'exopred'. The list of the in vitro exoproteome proteins was also inserted to the CpDB into a table called 'exo' that discriminates the identification of each protein regarding GenBank (protein id), as well as in which strains it is found. To make a relationship between the 'exopred' and 'exo' tables, a third table of the $\mathrm{CpDB}$, called 'gene', which contains all the functional annotation of the genomes of C. pseudotuberculosis, was created. The CpDB is the repository of the pan genome of C. pseudotuberculosis, harbouring the genomes since their initial genomic prediction, deposited in the GenBank, as well as the annotation corrections for future deposits. For this last purpose, the $\mathrm{CpDB}$ stores the identification of each protein according to the GenBank. In this way, it is possible to make a link between the three tables in the form of a clause of JOIN of the SQL: “... WHERE gene.locus_tag = exopred.locus_tag AND gene.protein_id $=$ exo.protein_id AND exopred.pangenome_coverage $=$ ' $5 x$ ' ...'. This clause returns the registries of the $\mathrm{CpDB}$ whose locus_tag in the gene table is equal to the locus_tag of the explored table, being this same gene in the protein_id field in the exo table with prediction of belonging to all five genomes. Other conditions can also be included, such as for example, restraining the results to specific genes of a C. pseudotuberculosis strain or simultaneously present in the exoproteome of specific strains.

\section{Additional material}

Additional file 1: C. pseudotuberculosis genomes. The five

C. pseudotuberculosis genomes here checked, as EMBL files.

Additional file 2: Predicted C. pseudotuberculosis pan secretome. List of the 150 genes for 750 locus_tags from the five C. pseudotuberculosis strains.

Additional file 3: Predicted C. pseudotuberculosis pan surfaceome. List of the 227 genes for 1135 locus_tags from the five C. pseudotuberculosis strains.

\section{Acknowledgements}

We would like to thank Daniel Restrepo-Montoya, Camilo Pino, Luis F Nino, Manuel E Patarroyo and Manuel A Patarroyo for their support with NGClass+ predictions. We would also like to thank to LISI, ALGOS-UN and FIDIC bioinformatics staff which were funded supported by Asociación Investigación Solidaria SADAR, Caja Navarra (CAN) (Navarra, Spain) and the Spanish Agency for International Development Cooperation (AECID). Funding: CNPq - Conselho Nacional de Desenvolvimento Científico e Tecnológico - Brazil.

This article has been published as part of BMC Genomics Volume 13 Supplement 5, 2012: Proceedings of the International Conference of the Brazilian Association for Bioinformatics and Computational Biology $(X-$ meeting 2011). The full contents of the supplement are available online at http://www.biomedcentral.com/bmcgenomics/supplements/13/S5.

\section{Author details}

${ }^{1}$ Molecular and Celular Genetics Laboratory, Instituto de Ciências Biológicas, Universidade Federal de Minas Gerais, Belo Horizonte, Minas Gerais, Brazil. 2DNA Polimorfism Laboratory, Universidade Federal do Pará, Campus do Guamá - Belém, PA, Brazil. ${ }^{3}$ Centre for Genomics and Applied Gene Technology, Institute of Integrative Omics and Applied Biotechnology, Nonakuri, Purba Medinipur, West Bengal, India. 


\section{Authors' contributions}

VA encouraged the research, BMC application, provided references, applied biological knowledge and gave final approval of the version to be published. ARS conducted all the software analyses, manually corrected annotation errors in the five genomes, developed the prediction schema and wrote the paper. EB contributed to the manually curation of all pseudogenes from all bacterial strains. MZT made substantial contributions to the design and interpretation of the manuscript. UP, AG, FD, FS, AC, AP, $D B, F F, L G, R R, S A, S S, V C A, A S$ and $A M$ have given final approval of the version to be published.

\section{Competing interests}

The authors declare that they have no competing interests.

Published: 19 October 2012

\section{References}

1. Rappuoli R: Reverse vaccinology. Curr Opin Microbiol 2000, 3:445-450.

2. Sibbald MJJB, van Dij JML: Secretome Mapping in Gram-Positive Pathogens. In Karl Wooldridge (ed.), Bacterial Secreted Protein: Secretory Mechanisms and Role in Pathogenesis. Caister Academic Press 2009, 193-225.

3. Simeone R, Bottai D, Brosch R: ESX/type VII secretion systems and their role in host-pathogen interaction. Curr Opin Microbiol 2009, 12:4-10.

4. Stavrinides J, McCann HC, Guttman DS: Host-pathogen interplay and the evolution of bacterial effectors. Cell Microbiol 2008, 10:285-292.

5. Bhavsar AP, Guttman JA, Finlay BB: Manipulation of host-cell pathways by bacterial pathogens. Nature 2007, 449:827-834.

6. Barinov A, Loux V, Hammani A, Nicolas P, Langella P, Ehrlich D, Maguin E, van de Guchte M: Prediction of surface exposed proteins in Streptococcus pyogenes, with a potential application to other Grampositive bacteria. Proteomics 2009, 9:61-73.

7. Strasser BJ: A world in one dimension: Linus Pauling, Francis Crick and the central dogma of molecular biology. Hist Philos Life Sci 2006, 28:491-512.

8. Rappuoli R: IS15 Developing vaccines in the era of genomics and toll receptors. Immunology 2005, 116:1

9. Rinaudo CD, Telford JL, Rappuoli R, Seib KL: Vaccinology in the genome era. J Clin Invest 2009, 119:2515-2525.

10. Lapierre P, Gogarten JP: Estimating the size of the bacterial pan-genome. Trends Genet 2009, 25:107-110.

11. Bambini S, Rappuoli R: The use of genomics in microbial vaccine development. Drug Discov Today 2009, 14:252-260.

12. Dorella FA, Pacheco LG, Seyffert N, Portela RW, Meyer R, Miyoshi A, Azevedo V: Antigens of Corynebacterium pseudotuberculosis and prospects for vaccine development. Expert Rev Vaccines 2009, 8:205-213.

13. Afzal M, Sakir M, Hussain MM: Corynebacterium pseudotuberculosis infection and lymphadenitis (taloa or mala) in the camel. Trop Anim Health Prod 1996, 28:158-162

14. Aleman M, Spier SJ, Wilson WD, Doherr M: Corynebacterium pseudotuberculosis infection in horses: 538 cases (1982-1993). J Am Vet Med Assoc 1996, 209:804-809.

15. Silva A, Schneider MPC, Cerdeira L, Barbosa MS, Ramos RTJ, Carneiro AR, Santos R, Lima M, D'Afonseca V, Almeida SS, Santos AR, Soares SC, Pinto AC, Ali A, Dorella FA, Rocha F, de Abreu VAC, Trost E, Tauch A, Shpigel N, Miyoshi A, Azevedo V: Complete genome sequence of Corynebacterium pseudotuberculosis 119 , a strain isolated from a cow in Israel with bovine mastitis. J Bacteriol 2011, 193:323-324.

16. Selim SA: Oedematous skin disease of buffalo in Egypt. J Vet Med B Infect Dis Vet Public Health 2001, 48:241-258.

17. Peel MM, Palmer GG, Stacpoole AM, Kerr TG: Human lymphadenitis due to Corynebacterium pseudotuberculosis: report of ten cases from Australia and review. Clin Infect Dis 1997, 24:185-191.

18. Trost E, Ott L, Schneider J, Schröder J, Jaenicke S, Goesmann A, Husemann P, Stoye J, Dorella FA, Rocha FS, Soares SDC, D'Afonseca V Miyoshi A, Ruiz J, Silva A, Azevedo V, Burkovski A, Guiso N, Join-Lambert OF, Kayal S, Tauch A: The complete genome sequence of Corynebacterium pseudotuberculosis FRC41 isolated from a 12-year-old girl with necrotizing lymphadenitis reveals insights into gene-regulatory networks contributing to virulence. BMC Genomics 2010, 11:728.

19. Ruiz JC, D'Afonseca V, Silva A, Ali A, Pinto AC, Santos AR, Rocha AAMC, Lopes DO, Dorella FA, Pacheco LGC, Costa MP, Turk MZ, Seyffert N,
Moraes PMRO, Soares SC, Almeida SS, Castro TLP, Abreu VAC, Trost E, Baumbach J, Tauch A, Schneider MPC, McCulloch J, Cerdeira LT, Ramos RTJ, Zerlotini A, Dominitini A, Resende DM, Coser EM, Oliveira LM, Pedrosa AL, Vieira CU, Guimarães CT, Bartholomeu DC, Oliveira DM, Santos FR, Rabelo EM, Lobo FP, Franco GR, Costa AF, Castro IM, Dias SRC, Ferro JA, Ortega JM, Paiva LV, Goulart LR, Almeida JF, Ferro MIT, Carneiro NP, Falcão PRK, Grynberg P, Teixeira SMR, Brommonschenkel S, Oliveira SC, Meyer R, Moore RJ, Miyoshi A, Oliveira GC, Azevedo V: Evidence for Reductive Genome Evolution and Lateral Acquisition of Virulence Functions in Two Corynebacterium pseudotuberculosis Strains. PLOS ONE 2011, 6:e18551.

20. Bendtsen JD, Nielsen H, Widdick D, Palmer T, Brunak S: Prediction of twinarginine signal peptides. BMC Bioinformatics 2005, 6:167.

21. Bendtsen JD, Kiemer L, Fausbøll A, Brunak S: Non-classical protein secretion in bacteria. BMC Microbiol 2005, 5:58.

22. Restrepo-Montoya D, Pino C, Nino LF, Patarroyo ME, Patarroyo MA: NClassG +: A classifier for non-classically secreted Gram-positive bacterial proteins. BMC Bioinformatics 2011, 12:21.

23. Petersen TN, Brunak S, von Heijne G, Nielsen H: SignalP 4.0: discriminating signal peptides from transmembrane regions. Nat Methods 2011, 8:785-786

24. Rutherford K, Parkhill J, Crook J, Horsnell T, Rice P, Rajandream MA, Barrell B: Artemis: sequence visualization and annotation. Bioinformatics 2000, 16:944-945.

25. Pacheco LGC, Slade SE, Seyffert N, Santos AR, Castro TLP, Silva WM, Santos AV, Santos SG, Farias LM, Carvalho MAR, Pimenta AMC, Meyer R, Silva A, Scrivens JH, Oliveira SC, Miyoshi A, Dowson CG, Azevedo V: A combined approach for comparative exoproteome analysis of Corynebacterium pseudotuberculosis. BMC Microbiol 2011, 11:12.

26. Deng LL, Humphries DE, Arbeit RD, Carlton LE, Smole SC, Carroll JD: Identification of a novel peptidoglycan hydrolase CWIM in Mycobacterium tuberculosis. Biochim Biophys Acta 2005, 1747:57-66.

27. Saxena A, Srivastava V, Srivastava R, Srivastava BS: Identification of genes of Mycobacterium tuberculosis upregulated during anaerobic persistence by fluorescence and kanamycin resistance selection. Tuberculosis (Edinb) 2008, 88:518-525.

28. Sun $L$, Zhang $L$, Zhang $H$, He Z: Characterization of a Bifunctional $\beta$ Lactamase/Ribonuclease and Its Interaction with a Chaperone-Like Protein in the Pathogen Mycobacterium tuberculosis H37Rv. Biochemistry (Moscow) 2011, 76:350-358.

29. Velmurugan K, Chen B, Miller JL, Azogue S, Gurses S, Hsu T, Glickman M, Jacobs WRJ, Porcelli SA, Briken V: Mycobacterium tuberculosis nuoG is a virulence gene that inhibits apoptosis of infected host cells. PLoS Pathog 2007, 3:e110

30. Zhang Z, Bulloch EMM, Bunker RD, Baker EN, Squire CJ: Structure and function of GImU from Mycobacterium tuberculosis. Acta Crystallogr D Biol Crystallogr 2009, 65:275-283.

31. McCarthy TR, Torrelles JB, MacFarlane AS, Katawczik M, Kutzbach B, Desjardin LE, Clegg S, Goldberg JB, Schlesinger LS: Overexpression of Mycobacterium tuberculosis manB, a phosphomannomutase that increases phosphatidylinositol mannoside biosynthesis in Mycobacterium smegmatis and mycobacterial association with human macrophages. Mol Microbiol 2005, 58:774-790.

32. Lin T, Melgar MM, Kurth D, Swamidass SJ, Purdon J, Tseng T, Gago G Baldi P, Gramajo H, Tsai S: Structure-based inhibitor design of AccD5, an essential acyl-CoA carboxylase carboxyltransferase domain of Mycobacterium tuberculosis. Proc Natl Acad Sci USA 2006, 103:3072-3077.

33. Jones $C M$, Niederweis M: Mycobacterium tuberculosis can utilize Heme as an iron source. J Bacteriol 2011, 193:1767-1770.

34. Davies MN, Flower DR: Harnessing bioinformatics to discover new vaccines. Drug Discov Today 2007, 12:389-395.

35. Santos A, Ali A, Barbosa E, Silva A, Miyoshi A, Barh D, Azevedo V: The reverse vaccinology - A contextual overview. IIOABJ 2011, 2:8-15.

36. Azevedo V, Santos AR, Soares S, Ali A, Pinto A, Magalhaes A, Barbosa E, Ramos R, Cerdeira L, Carneiro A, Abreu V, Almeida S, Schneider P, Silva A, Miyoshi A: Automated functional annotation. In Bioinformatics - trends and methodologies. Volume 1. InTechOpen;Mahdavi MA 2011:722.

doi:10.1186/1471-2164-13-S5-S6

Cite this article as: Santos et al:: The Corynebacterium pseudotuberculosis in silico predicted pan-exoproteome. BMC Genomics 2012 13(Suppl 5):S6. 